\title{
A SYSTEM FOR DEPOSITING TUNGSTEN COATINGS SUITABLE FOR AN ELECTRICAL CONTACT APPLICATION
}

\author{
R. CARPENTER \\ Allen Clark Research Centre, The Plessey Company Limited, Caswell, Towcester, Northants, U.K.
}

(Received August 7, 1975)

\begin{abstract}
A method was needed for depositing smooth coatings of tungsten at relatively high rates onto the electrodes of a reed switch. A literature survey indicated that the most suitable technique was based on the vapour phase hydrogen reduction of tungsten hexafluoride. A reactor was designed for this purpose on the basis of safety, optimum utilisation of tungsten hexafluoride, and possible adaption to higher throughput. Experience with this reactor indicated that precautions were necessary before good quality coatings could be deposited and suggested that films of an acceptable thickness variation could be produced by using a flow reversal technique.
\end{abstract}

\section{INTRODUCTION}

A contact material was required for the development of a reed switch. The switch of novel design employed magnetic coupling through the contact material itself and needed to handle currents of a few amperes at open circuit potentials in excess of 100 volt. Tungsten contacts sealed in an oxygen-free environment offered the best solution, but restrictions on the permissible thickness were imposed by the coupling requirement. There was a maximum thickness which could be used and yet still give a sufficient closure force with an acceptable number of ampere-turns in the energising coil. On the other hand, too thin a contact would lead to considerable shortening of contact life. These restrictions led to an acceptable band of thickness of between $30 \mu \mathrm{m}$ and $50 \mu \mathrm{m}$.

Discrete contacts as thin as $50 \mu \mathrm{m}$ were not readily available in the right form, as they needed to be cut from swaged rod to give a grain orientation to resist spalling. The other possibility, and the one examined in detail, was to deposit a coating of tungsten on the switch electrode (substrate) in such a way that the deposition on non-contact areas was minimised. The contact area was half of one face of a rectangular softiron substrate $20 \mathrm{~mm} \times 5 \mathrm{~mm}$.

Bearing in mind possible future development, it was estimated that in any experimental deposition system at least one hundred substrates would have to be coated in a batch at a rate of $5 \mu \mathrm{m}$ to $10 \mu \mathrm{m}$ per min. A brief survey of possible deposition techniques was made to find the one most suitable for this application. Having done this, an experimental system for depositing tungsten was constructed.

\section{TECHNIQUES FOR DEPOSITING TUNGSTEN COATINGS}

There are very few suitable methods. Vacuum deposition techniques did not seem promising on account of the temperature required to give the high deposition rate. Published work ${ }^{1-3}$ using laboratory scale equipment for metallisation refers to rates below $0.05 \mu \mathrm{m} / \mathrm{min}$. A source temperature exceeding $3,300 \mathrm{~K}$ would be required for evaporation and although this should be possible with an electronbombardment heated source, the cost of developing a suitable system would be considerable. Similarly, low rates have been reported for tungsten sputtering ${ }^{4}$ although recent advances in sputtering systems ${ }^{5}$ offer the prospect of considerable improvement.

Electrodeposition techniques appeared to be equally inapplicable. Early work by Davis ${ }^{6}$ showed that it was impossible to deposit tungsten from an aqueous solution, as the electrode potential needed lies far beyond that needed by hydrogen so that water is always decomposed first of all. Deposition from organic solutions of tungsten salts also proved to be impossible. However, the metal was electrodeposited from mixed borate baths fused at $1150-1200 \mathrm{~K}$, but severe corrosion problems were encountered with the salts and their reaction products which had to be overcome by shielding the exposed metal parts with a flow of nitrogen. Rates of up to $25 \mu \mathrm{m} / \mathrm{min}$. and deposits up to $500 \mu \mathrm{m}$ thick were quoted. Other workers ${ }^{7,8}$ have reported on electrodeposition from molten salts, but Brenner ${ }^{9}$ has cast some doubt on the thickness uniformity and surface finish of the tungsten coatings obtained. 
Arc-plasma spraying techniques for depositing tungsten have been developed on a commercial basis, but little detailed information has been published ${ }^{10}$ and no firm assessment of the viability of coatings for contacts could be made. The method involves blowing tungsten powder through an arc struck in a non-oxidising environment and the granularity of the deposit depends on the mesh size of the powder. Very fine powders tend to be scattered easily from the jet stream and without recycling the powder the process becomes inefficient. However, recycling leads to an increase in the impurity level of the deposit. These factors would all be important for the contact application.

Chemical vapour deposition (C.V.D.) is used to deposit a variety of films. General articles have been published by Feist ${ }^{11}$ and Amick ${ }^{12}$, the latter with particular reference to films used in microelectronics. A number of tungsten-forming reactions are possible. The metal can be deposited directly by pyrolytically decomposing the carbonyl ${ }^{13}$ at $1,200 \mathrm{~K}$, but the deposition rate is low and any attempt to raise the temperature leads to a gas-phase reaction and a loose porous deposit. The other C.V.D. techniques depend on reactions of tungsten halides. A de-Boer type reaction has been used with the bromides ${ }^{14}$ in which bromides were formed from the elements at $1,100 \mathrm{~K}$ and deposited at $1,800 \mathrm{~K}$. The pressure was reduced to promote the tungsten-forming reaction. Provided that care was taken to exclude oxygen, uniform adherent deposits up $6 \mathrm{~mm}$ thick could be produced. A similar reaction can be used with the iodides ${ }^{15}$, but the substrate temperature needed is impracticably high. In the case of the chloride ${ }^{16}$ the halide forming reaction occurs at only $700 \mathrm{~K}$, but the substrate temperature of $1700 \mathrm{~K}$ to $1900 \mathrm{~K}$ is too high for iron substrates. A much easier technique is to employ the hydrogen reduction of the hexachloride ${ }^{17}$, where dense adherent deposits are formed with the substrates at $850 \mathrm{~K}$ to $950 \mathrm{~K}$. Glaski ${ }^{18}$ has used chlorination of tungsten followed by hydrogen reduction to achieve the same result, but with both reactions occurring in one plant. The tungsten chips were cleaned in situ by firing in hydrogen before chlorination at $1300 \mathrm{~K}$.

The hexafluoride, instead of the hexachloride, can be used as the starting material to considerable advantage. ${ }^{9}$ The reaction

$$
\mathrm{WF}_{6}+3 \mathrm{H}_{2}=\mathrm{W}+6 \mathrm{HF}
$$

has been used in most recently reported work on tungsten vapour deposition ${ }^{19-22}$ and there is general agreement that it offers advantages over the other techniques described above. The substrate temperature required to achieve good coherent deposits is only $800 \mathrm{~K}$ and deposit rates of a few microns per minute are obtained easily. The reaction can be carried out at atmospheric pressure and the low boiling point, $292.5 \mathrm{~K}$, of the fluoride means that it is easy to maintain a sufficient supply of vapour to the reactor. An advantage cited by Berkeley ${ }^{20}$ is the absence of more stable lower fluorides to interfere with the homogeneity of the tungsten deposit, although Glaski ${ }^{2}$ has reported mass spectrometric evidence for the presence of tungsten tetra-fluoride (B.P. $900 \mathrm{~K}$ ) in the tungsten. The deposited tungsten shows a columnar structure 20,24 with major grain boundaries running perpendicular to the substrate; this type of structure $^{25}$ is most resistant to spalling of the tungsten which can occur following temporary welding of the switch contacts. A possible limitation is the adhesion of the film to iron substrates, since according to Bryant ${ }^{26}$ the behaviour is inconsistent. However, since tungsten adheres well to both copper and nickel, this problem could be overcome by electroplating the contacts first of all.

Of all the methods considered for tungsten deposition the easiest and most well documented was evidently the C.V.D. fluoride technique and as this also seemed adaptable to the contact application it was selected for the experimental study.

\section{DESIGN OF C.V.D. SYSTEM}

In the design of the experimental system and its possible scaling to a production sized facility, two considerations needed particular attention. Foremost of these was that of safety, since both tungsten hexafluoride and the reaction by-product hydrogen fluoride are obnoxious and are mixed with potentially explosive hydrogen. Secondly, although tungsten fluoride is readily available commercially, the cost of the tungsten is 2 to 4 times that of the equivalent metal contained within it.

Initially a choice had to be made between a static and a continuous flow deposition system. Two factors limit the amount of tungsten available for deposition in a static system, the mass of the tungsten fluoride and the equilibrium of the reaction. According to Berkely, ${ }^{20}$ the reaction in a static volume is inhibited by a hydrogen fluoride concentration above $25 \%$ of the total. Typically rather more than the stoichiometric volume ratio is used, so that it is readily shown that to deposit a $30 \mu \mathrm{m}$ coating on the contact area of 


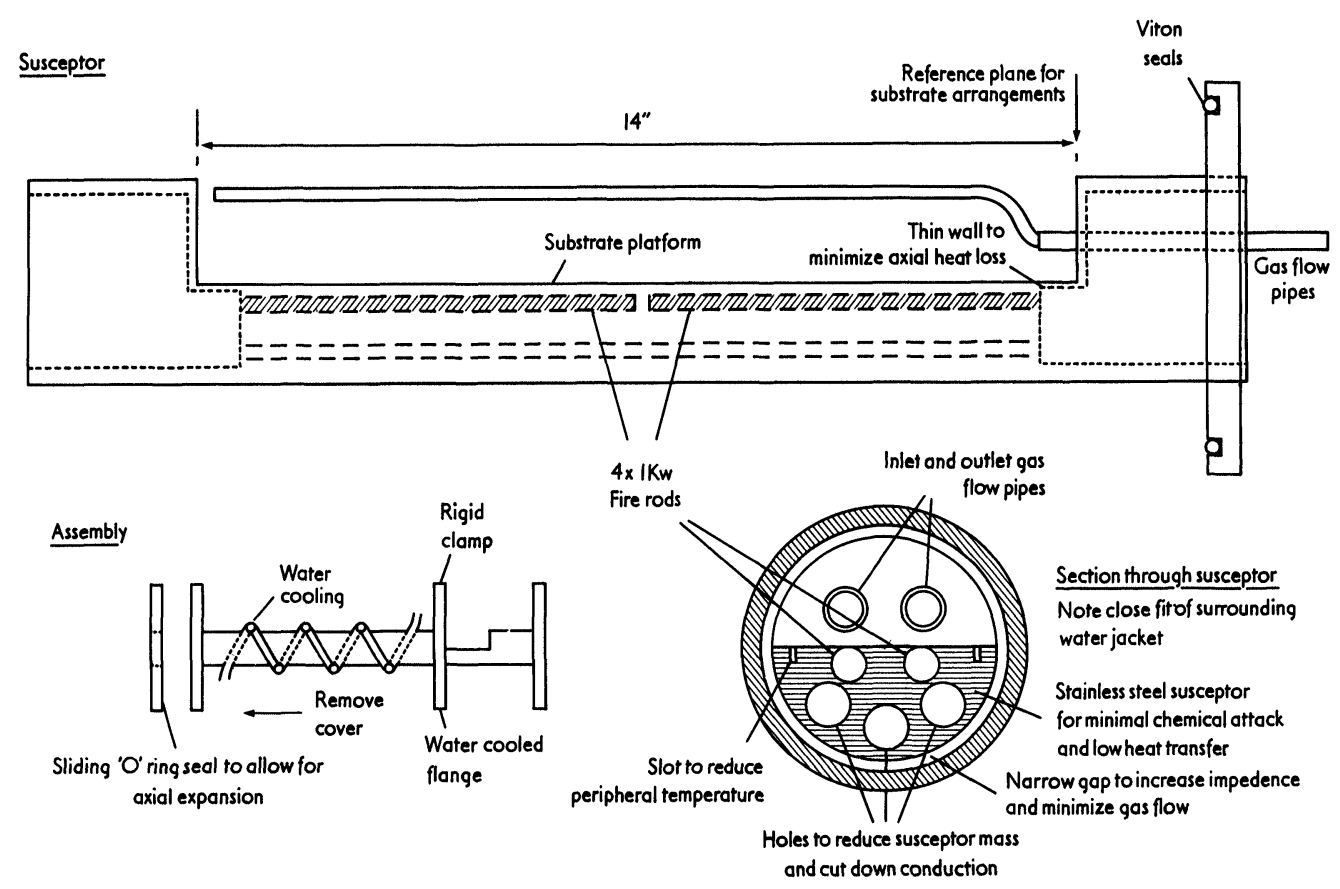

FIGURE 1 Apparatus for C.V.D. of tungsten from WF 6

$50 \mathrm{~mm}^{2}$ with an efficiency of $50 \%$ requires in excess of $100 \mathrm{~cm}^{3}$ of gas. Hence the reactor volume necessary to coat upward of hundred contacts in a static system is excessively large and would be awkward to fill and empty. It appeared to be much more advantageous to use a continuous flow system, where the concentrations of the reactants can be easily controlled.

Published results ${ }^{20}$ indicate that the optimum deposition temperature is $\sim 800 \mathrm{~K}$, since higher temperatures lead to a gas-phase reaction and the formation of a loose non-adherent deposit. All surfaces except the contact areas need to be kept cool for maximum tungsten utilisation, designs in which the whole deposition chamber is heated are therefore uneconomic. Direct and indirect radio-frequency heating of the substrates were considered but rejected; the former on account of the difficulty of simultaneously coupling power into a large number of substrates, the latter since an R.F. heated susceptor offered no significant advantage over a substrate holder heated with resistive elements.

The most convenient reactor geometry to use with a continuous flow system is one based on cylindrical symmetry. Ideally, the substrates should be mounted around the inside of a heated tube, along which the reactants pass, to minimise deposition on the noncontact areas. Alternatively, the substrates could be mounted around the periphery of a heated core.

Mounting the substrates so as to maximise deposition on the contact areas presents difficulties whichever method is chosen and a compromise between these possibilities and an arrangement which would simplify loading was adopted.

This design, which consists of a heated core enclosed within a cooled jacket, is illustrated in Figure 1. Besides enabling the substrates to be loaded easily, the arrangement gives reasonable temperature uniformity along the substrate-bearing surface whilst maintaining the other surfaces as cool as possible. All the main parts are fabricated in stainless steel to offer the best corrosion resistance and to provide high thermal impedance.

The substrate-bearing surface is formed by milling the central part of a cylindrical bar to a semicircular section. Two $1 \mathrm{kw}$ cartridge heaters are inserted at each end into two holes drilled paraxially, close to the substrate-bearing surface. Each pair of heaters is controlled independently. A number of other paraxial holes serve to reduce the thermal mass and increase the radial thermal impedance besides permitting forced-air cooling to be used to cool the outer 


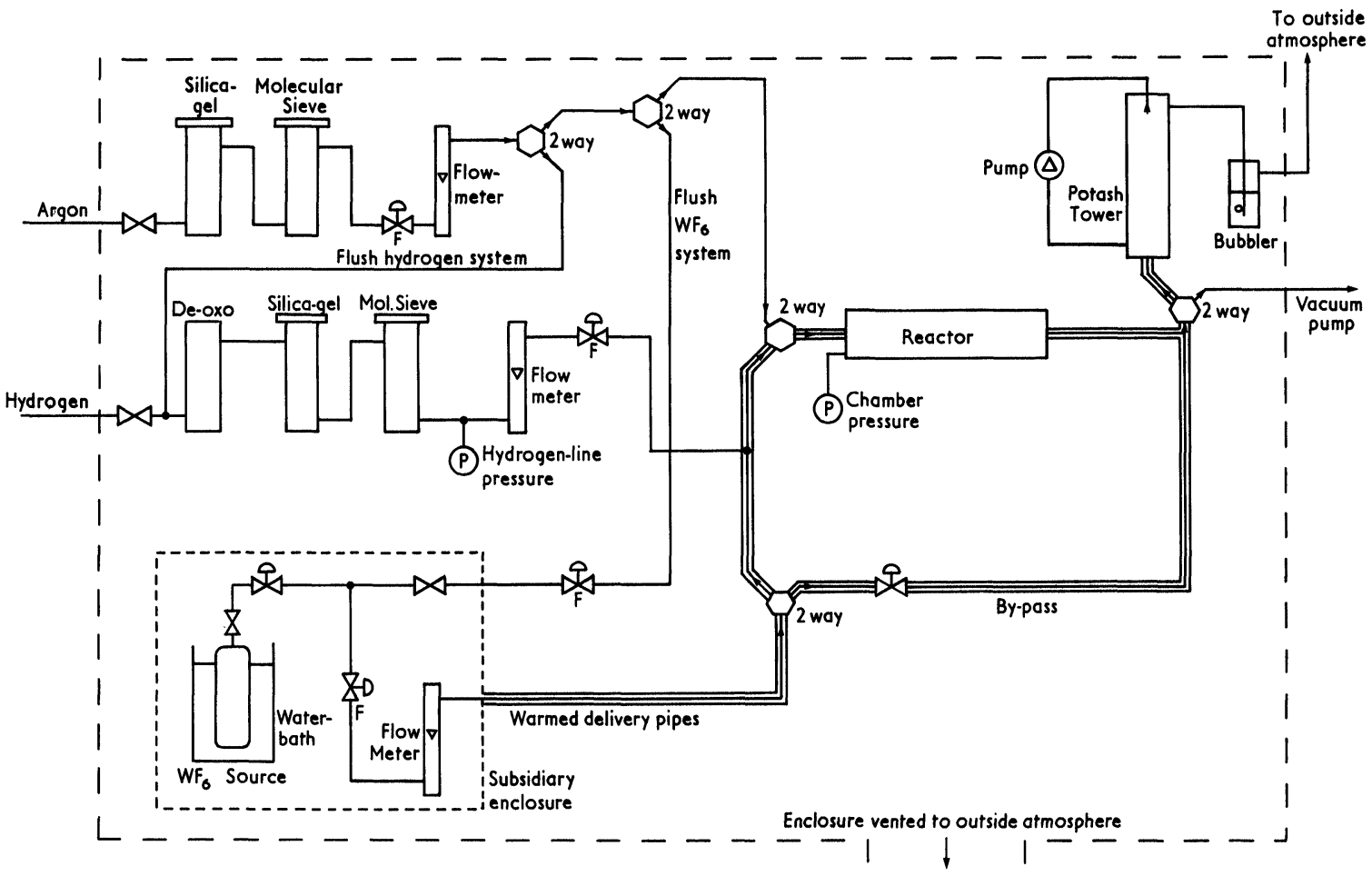

FIGURE 2 Overall layout of deposition system

surfaces of the bar. A thermocouple inserted through one of these holes into the centre of the bar provides a convenient method of establishing a temperature reference. At the ends, the wall thicknesses are reduced to improve thermal isolation of the core. A demountable and close fitting water-cooled jacket concentric with the core forms the reactor volume. Flanges welded to the core and water-cooled jacket in conjunction with a 'Viton' O-ring form a hermetic seal at one end; while a standard sliding seal, formed by an O-ring sealing on the periphery of the core and retained by a free flange and flange welded to the water-cooled jacket, allows for axial expansion and subsequent contraction at the other. The close fit of the water-cooled jacket surrounding the core restricts the gas flow and hence extraneous tungsten deposition on the periphery.

This construction has certain other advantages. Both the gas and electrical connections can be made entirely through the flanged end of the core, so that the jacket can be completely removed to give access for loading and unloading the substrates. The heaters and their associated electrical connections are completely isolated from the reactor volume itself and the chance of explosion from accidental electrical discharge is eliminated. Furthermore, since the volume of gas exposed at any instant within the reactor is small, the hazard from explosion is kept to a minimum.

Safety is also a primary requirement for the gas distribution system. Proprietary stainless steel fittings and tubulation are consequently used throughout in the reactant gas lines. Figure 2 shows a schematic diagram. The flows of both hydrogen and tungsten hexafluoride are monitored and can be adjusted with needle valves. The hydrogen is obtained from a cylinder situated well away from the reactor. The high-purity cylinder gas is passed through a deoxo unit and then into series-connected drying towers containing self-indicating silica-gel and molecular sieves. The tungsten hexafluoride is obtained from a lecture bottle, which is supported in a thermostatically controlled low-temperature water-bath. The control valve and flowmeter are positioned immediately adjacent to the water-bath and in the same enclosure to keep their temperatures above $293 \mathrm{~K}$ and prevent liquefaction of the hexafluoride gas. All pipework containing tungsten hexafluoride is held at a temperature above $293 \mathrm{~K}$ by heaters. These are widely spaced turns of nichrome wire insulated 
from the pipes and held in position with P.T.F.E. tape and are operated from appropriate secondary tappings of a low-voltage heater transformer.

The hydrogen and tungsten hexafluoride mixture is normally fed directly into the reactor and the reaction products are then passed into the absorption tower to remove fluorides. The tower contains a continuously recirculating solution of potassium hydroxide, which is sprayed onto the top of closely packed lengths of P.V.C. tubing through which the gases diffuse upward. It is designed to prevent accidental suck-back of the solution into the hot reactor. Finally, the hydrogen is vented directly into the atmosphere outside the laboratory.

The entire system can be flushed out with argon to expel all traces of reactants and their gaseous products. Like the hydrogen, the argon is thoroughly dried before use. Capsule-type pressure gauges are fitted both to the hydrogen line and to the chamber volume to ensure that no excessive pressures are built up. A facility is also provided to enable rough evacuation of the chamber after the reactor has been exposed to the atmosphere; this assists in the removal of traces of water.

Finally the reactor and all the gas lines are contained within a fume cupboard, which is formed in perspex and is fitted with a 'blow out' panel situated away from the operator. The extractor fan for the fume cupboard is interlocked with the reactor heaters to ensure that it is always operating during tungsten deposition.

\section{DEPOSITION PROCEDURE}

The nickel-coated iron substrates are arranged on the substrate platform (Figure 1) so that the contact surfaces alone are exposed to the main gas stream. This is achieved by using an echelon arrangement with each succeeding substrate masking the non contact area of the preceeding one. Five rows of substrates, stacked close together to minimise gas flow to the under-surface and each containing 25 substrates, are used. The plane of each substrate subtends an angle of $\sim 10^{\circ}$ with the substrate platform and the exposed contact surfaces face toward the gas inlet. After assembling the water-cooled jacket, the entire system is flushed out with argon. The reactor is next isolated and evacuated with the rotary pump, while the heaters are set to a predetermined level to raise the core temperature to $500 \mathrm{~K}$. The pump is isolated, the heater power increased and a hydrogen-argon flow introduced into the reactor.
The argon is added through the tungsten hexafluoride line and serves to reduce the thermal conductivity of the gas. When hydrogen is used by itself, the rapid rise in temperature occurring on introducing tungsten hexafluoride leads to non-reproducible deposition conditions. By choosing the right flow for the argon it is possible to ensure that little or no change in the reactor temperature occurs when tungsten hexafluoride is admitted. After the temperature has stabilised at about $750 \mathrm{~K}$, the argon supply is shut off and tungsten hexafluoride is introduced. Typically the flow rates are $1.21 / \mathrm{min}$ for hydrogen and $0.151 / \mathrm{min}$. for tungsten hexafluoride with $62.5 \mathrm{~cm}^{2}$ of contact surface. After the required thickness has been deposited, both the tungsten hexafluoride supply and the reactor heaters are switched off. The hydrogen flow through the reactor is maintained to promote rapid cooling. When the temperature has fallen below $400 \mathrm{~K}$, the tungsten hexafluoride remaining in the pipeline is flushed through the reactor by-pass into the absorption tower while the hydrogen is kept flowing to prevent a back-flow into the reactor. Subsequently, the entire system is flushed through with argon to remove all traces of tungsten hexafluoride and hydrogen fluoride before the reactor is opened. Using this procedure, contamination-free tungsten films are produced.

\section{RESULTS}

The parameters of particular interest are the thickness and quality of the tungsten coatings and the utilisation of the tungsten hexafluoride.

Early experiments confirmed the observations of Bryant ${ }^{2} 7$ that tungsten films deposited directly on iron were generally non-adherent. However, with a nickel intermediate layer, the adhesion is high and the quality good as long as the temperature is below $800 \mathrm{~K}$. Coatings deposited at rates up to $5 \mu \mathrm{m} / \mathrm{min}$. are generally of excellent quality. The effects of imperfections in the underlying nickel layer are exaggerated by the mode of growth of the tungsten and it is essential for the underlying layer to be of good quality. As the coating thickness increases, the surface of the tungsten becomes rougher as a result of preferential grain growth.

Examination of coatings was carried out by preparing metallographic sections for viewing with an optical microscope and by means of a scanning electron microscope (Stereoscan). Selective etching of polished metallographic sections was achieved by using a nitric acid-methanol mixture for iron and a 


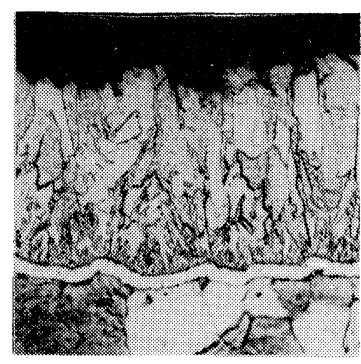

(a)

Section of typical coating

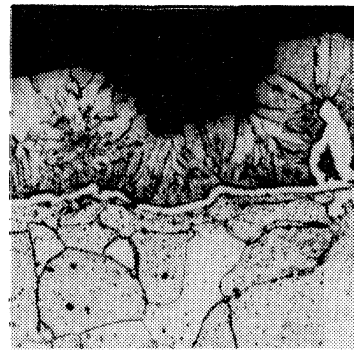

(c)

Nodular growth produced by underlayer defect

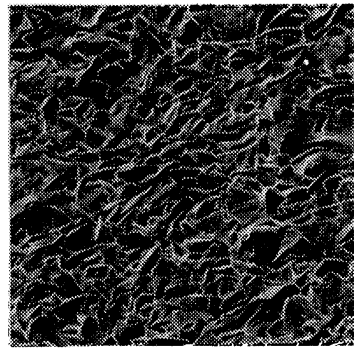

(e)

Typical

topography

potassium hydroxide-potassium ferricyanide solution for tungsten. Figure 3 shows examples of the various coatings together with some of the faults encountered. Figure $3(\mathrm{a})$ is a section through a thick tungsten coating and illustrates preferred grain growth and progressive grain enlargement. Figure 3(b) shows the presence of tungsten oxides (tungsten blue) formed at the nickel interface when the precautions taken to dry out the system before admitting tungsten hexafluoride were inadequate. The effect of an imperfection in the nickel underlayer which leads to the nodule formation on the surface of the tungsten is

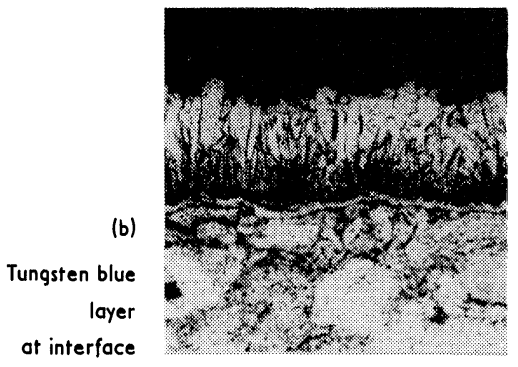

(d)

Nodule at surface
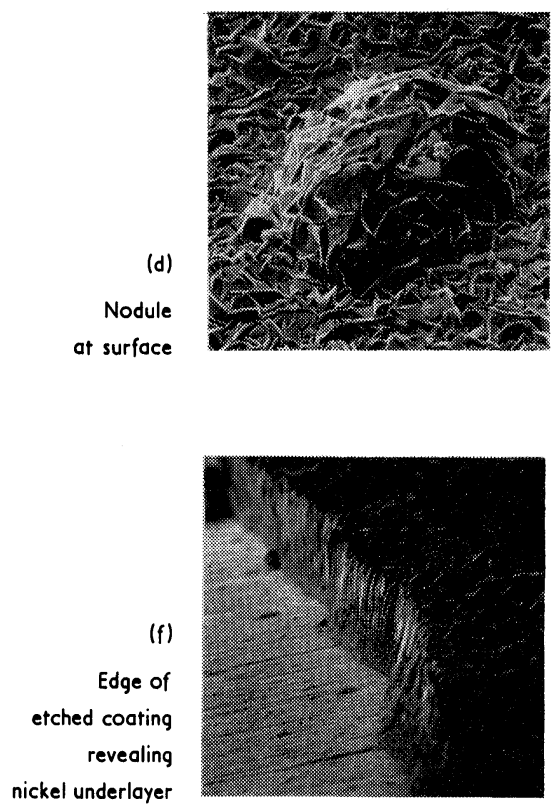

$40 \mu \mathrm{m}$

FIGURE 3 Tungsten coatings illustrated in Figure 3(c). Nodule formation can also be seen in the Stereoscan micrograph of Figure 3(d), while 3(e) shows the surface of a typical contact coating. Figure $3(\mathrm{f})$ is an edge of the coating produced by covering part of the deposit with a protective layer and then removing the unprotected portion with an anodic etch. The columnar structure of the film is clearly seen.

Two methods were used to estimate coating thickness. A time-consuming technique was to etch through parts of the coating to the substrate and then make direct measurements with a 'Talysurf' surface 


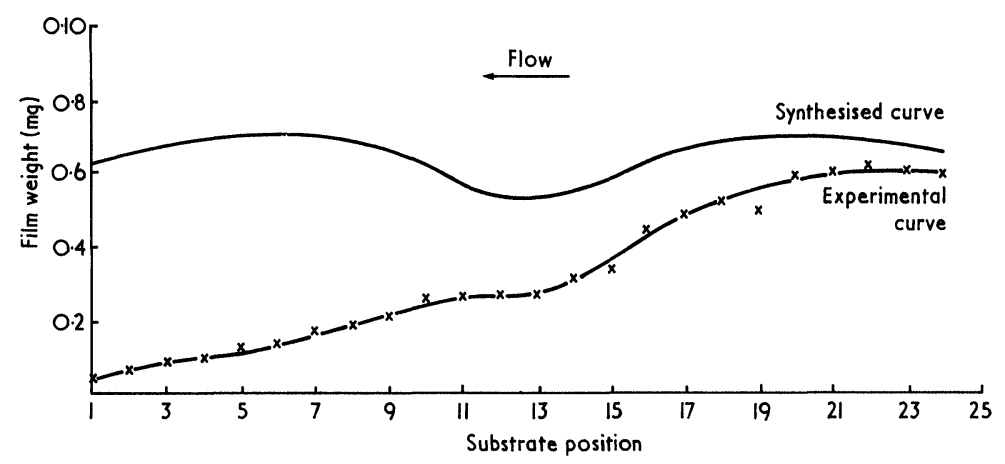

FIGURE 4 Substrate weight increase as a function of position.

profile monitor. A more convenient method was to weigh individual films. This was meaningful since identical areas of the substrates were exposed and 'Talysurf' measurements indicated that thickness variations over individual substrates were less than 5\%.

The thickness of tungsten deposited on individual substrates depended on the substrate position as well as the deposition parameters. Within a batch, consisting of 5 rows each containing 25 substrates, thickness variations could be minimised to $\pm 15 \%$ by using low temperatures and long deposition times. This minimised the depletion of tungsten hexafluoride in the gas stream, but yielded very low utilisation efficiencies. Economically, this would be unacceptable for a production technique. A more profitable approach is to maximise the tungsten utilisation, so that the coating thickness diminishes along the reactor. This is shown in Figure 4, where the weight of the coating deposited on each of 25 substrates is plotted against axial position. Each substrate is five times the width of those used for the contact application and its position is numbered from the gas inflow. A way of producing a more uniform thickness distribution is to arrange to reverse the direction of gas flow midway through the deposition process. However, this experiment could not be carried out with the existing reactor. The uniformity which might be expected from flow reversal can be inferred and is also plotted in Figure 4; the synthesised curve was obtained by adding the experimental curve to its mirror image in a plane through the centre and shows variations of thickness within $\pm 10 \%$. In practice it may be impossible to achieve this degree of uniformity without manipulating the substrates, because of the asymmetry relative to gas flow of echelon stacking. The tungsten utilisation efficiency was calculated from the total weight of the tungsten coatings on the contact areas and the measured weight loss of the tungsten coatings on the contact areas and the measured weight loss of the tungsten hexafluoride source cylinder. Expressed as percentages, $40 \%$ of the tungsten was deposited on the contact areas and $7 \%$ on the backs and edges of the substrates. The tungsten conversion efficiency was deduced by determining the tungsten residue was found by oxidising the fluid with hydrogen peroxide and acidifying with hydrochloric acid to precipitate tungsten oxide. The result indicated a conversion efficiency of $90 \%$. Thus about $40 \%$ of the tungsten is wasted through deposition on the hot parts of the reactor.

\section{CONCLUSIONS}

The literature survey indicated conclusively that the most satisfactory method for depositing thick tungsten films at high rates for the required contact application involved the use of the hydrogen reduction of tungsten hexafluoride.

A system designed to produce small numbers of contacts for evaluation purposes has proved to be remarkably simple to use and could be scaled up to produce a larger throughput without too much difficulty. In designing equipment for this purpose, the safety and utility of tungsten were aspects which were recognised initially. The experimental work indicated the need to start off with a smooth substrate surface, since any irregularity is emphasised by the growth mode, and the necessity of removing all traces of moisture.

The particular configuration of the contact does not lend itself to highly efficient tungsten utilisation. However, the results indicated that by using a system 
in which the direction of tungsten hexafluoride flow could be reversed it should be possible to achieve tungsten utilisation efficiencies of up to $40 \%$.

The method yields contacts both of acceptable smoothness for thicknesses up to $50 \mu \mathrm{m}$ and of suitable grain orientation, and could offer an alternative to wrought tungsten contacts in some applications.

\section{ACKNOWLEDGEMENTS}

The Author is indebted to Mr. G. V. Smith for the Stereoscan micrographs and Mr. J. Rigby for the chemical analysis. He would also like to thank the Directors of the Plessey Company Limited for permission to publish this paper.

\section{REFERENCES}

1. N. J. Maskalich and C. W. Lewis, Some Properties of Evaporated Refractory Metal Films, Trans. 8th Am. Vac. Symp. 2, 874 (1961).

2. F. L. Schuermeyer, W. R. Chase and E. L. King, Ion Effects During e-Beam Deposition of Metals, J. Vac. Sci. \& Technol. 9, 330 (1972).

3. A. K. Sinha, T. E. Smith, T. T. Sheng and N. N. Axelrod, Control of Resistivity in Electron Beam Evaporated Tungsten Films, J. Vac. Sci. \& Technol. 10, 436 (1973).

4. W. W. Y. Lee, High Resistivity of d.c. Sputtered Metal Films, J. Appl. Phys. 42, 4366 (1971).

5. R. L. Cormia, P. S. McLeod and N. K. Tsujimoto, High Rate Sputtering Achieved by Permanent Magnets, J. Electrochem. Soc. 121, 110C (1974).

6. G. L. Davis and C. H. R. Gentry, The Electrodeposition of Tungsten, Metallurgia 53, 3 (1956).

7. F. X. McCawley, C. B. Kenahan and D. Schlain, Electrodeposition of Tungsten Coatings from Molten Salts, J. Electrochem. Soc. 110, 180C (1963).

8. G. W. Mellors and S. Senderoff, The Electroforming of Refractory Metals, Plating 51, 972 (1964).

9. A. Brenner and W. E. Reid, Vapour Deposition of Tungsten, U.S. Patent No. 3,072,983 (1963).

10. A. R. Moss and W. J. Young, Arc Plasma Spraying, Science and Technology of Surface Coatings, ed. B. N. Chapman and J. C. Anderson (Academic Press 1974) p. 287.
11. W. M. Feist, S. R. Steele and D. W. Readey, The Preparation of Films by Chemical Vapour Deposition, Physics of Thin Films, ed. G. Hass and R. E. Thun Academic Press (1969) p. 237.

12. J. A. Amick and W. Kern, Chemical Vapour Deposition Techniques for the Fabrication of Semiconductor Devices, Proc. of 2nd Int. Conf. on C.V.D. (Electrochem. Soc. 1970) p. 551.

13. H. E. Carlton and W. M. Goldberger, Fundamental Considerations of Carbonyl Technology, J. Met. 17, 611 (1965).

14. R. M. Caves, Tungsten Coatings from the Thermal Deposition of Tungsten Bromides, Trans. A.I.M.E. 224, 267 (1962).

15. J. A. Moore and C. M. Jolly, Quartz Iodine Lamps, G.E.C. Journal 29, 99 (1962).

16. C. F. Powell, J. H. Oxley and J. M. Blocher, Vapour Deposition Electrochem. Soc. 1966 chap. 10, p. 322.

17. E. J. Mehalchick and M. B. MacInnis, Preparation of Vapour-Deposited Tungsten at Atmospheric Pressure, Electrochem. Technol. 6, 66 (1968).

18. F. A. Glaski, Controlling Grain Orientation in C.V.D. Tungsten, Proc. 2nd Int. Conf. on C.V.D. (Electrochem. Soc. 1970) p. 839.

19. V. A. Nieberlein, Vapour Deposited Tungsten Coatings on Graphite, Am. Ceram. Soc. Bull. 44, 14 (1965).

20. J. F. Berkeley, A. Brenner and W. E. Reid, Jr., Vapour Deposition of Tungsten by Hydrogen Reduction of Tungsten Hexafluoride, J. Electrochem. Soc. 114, 6 (1967).

21. A. M. Schroff and G. Delval, Recent Developments in the Chemical Vapour Deposition of Tungsten and Molybdenum, High Temp.-High Pressures 3, 695 (1971).

22. W. A. Bryant and G. H. Meier, Kinetics of the Chemical Vapour Deposition of Tungsten, J. Electrochem. Soc. 120, 559 (1973).

23. F. A. Glaski, The Use of Oxygen Additive to Control Residual Fluorine in Chemical Vapour Deposited Tungsten, Record of 9th Thermionic Specialist Conference (I.E.E.E. 1970) p. 72.

24. R. K. Chuzhko, I. V. Kirillov, Yu N. Golovanov and A. P. Zakharov, Texture of Tungsten Formed by Deposition from the Vapour, J. of Crystl. Growth 3, 219 (1968).

25. C. J. Smithels, Tungsten (Chapman and Hall 1945) chap. 11, p. 229.

26. W. A. Bryant, The Adherence of Chemically Vapour Deposited Tungsten Coatings, Proc. of 2nd Int. Conf. on C.V.D. (Electrochem. Soc. 1970) p. 409.

27. A. I. Vogel, Quantitative Inorganic Analysis (Longmans 1962) p. 567. 

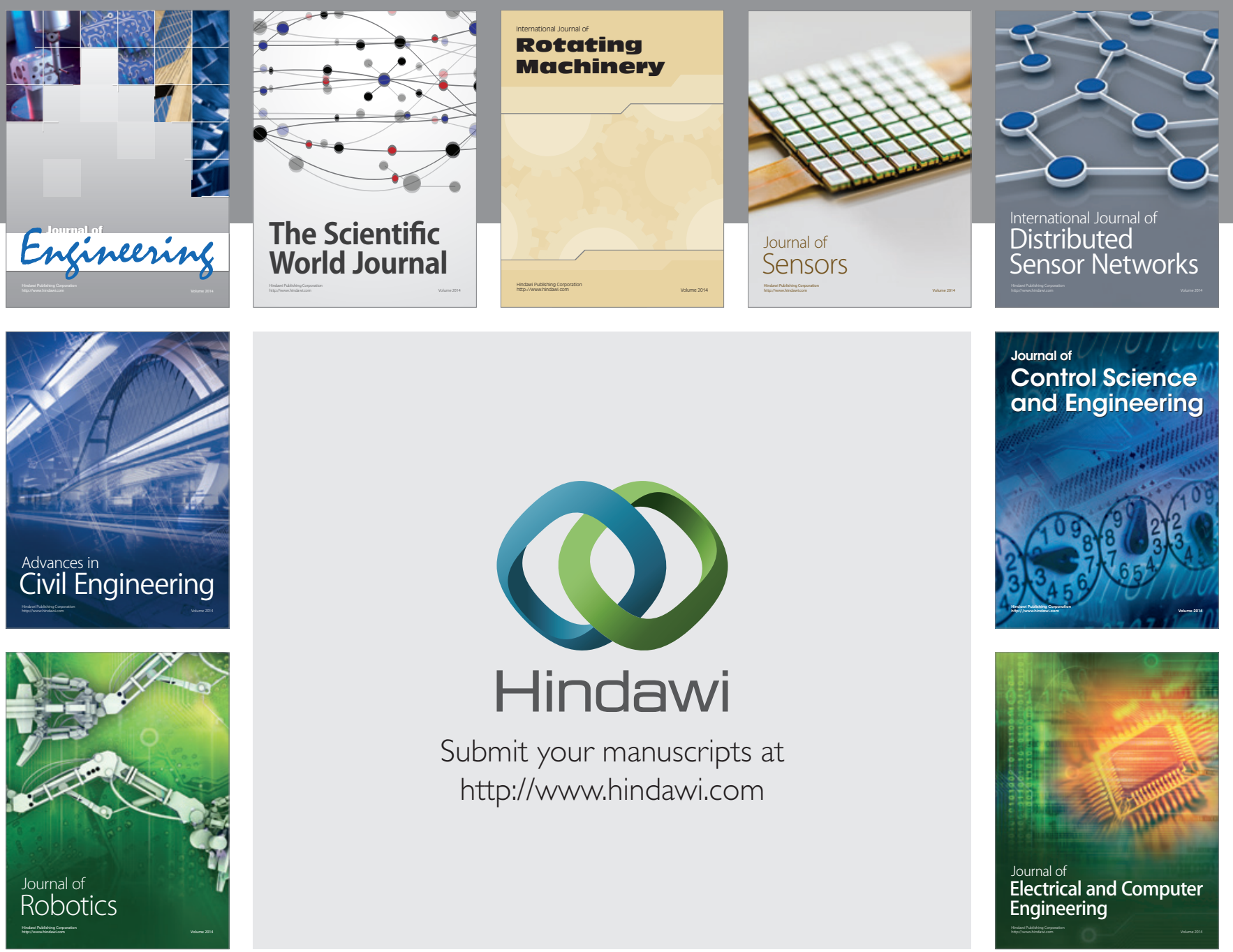

Submit your manuscripts at

http://www.hindawi.com
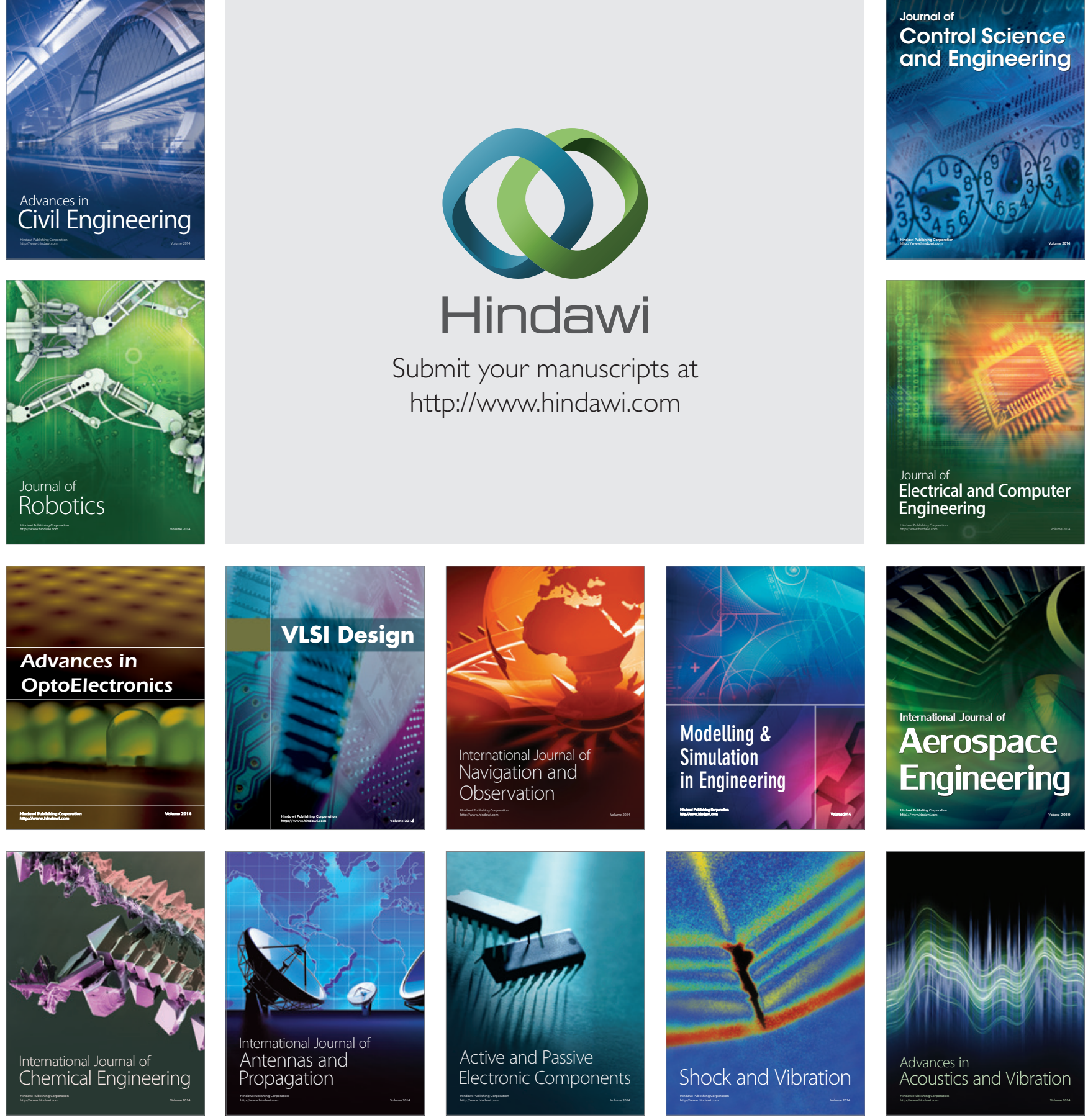